Çukurova Üniversitesi Mühendislik Mimarlık Fakültesi Dergisi, 32(3), ss. 9-18, Eylül 2017

Çukurova University Journal of the Faculty of Engineering and Architecture, 32(3), pp. 9-18, September 2017

\title{
Antakya-Cilvegözü Karayolu Etrafındaki Tarım Arazilerinde ve Bitkilerdeki Ağır Metal Kirliliği
}

\author{
Abdullah ÖZKAN ${ }^{* 1}$ \\ ${ }^{I}$ İskenderun Teknik Üniversitesi, Mühendislik ve Doğa Bilimleri Fakültesi, Petrol ve Doğalgaz \\ Mühendisliği Bölümü, Hatay
}

Geliş tarihi: 17.02.2017 Kabul tarihi: 25.09.2017

\section{$\ddot{\mathbf{O z}}$}

Bu çalışmada, Hatay İline bağlı Antakya-Cilvegözü uluslararası karayolu çevresindeki tarım arazilerinin ve bu arazilerde yetişen bitkilerin trafik ve tarımsal faaliyet kaynaklı ağır metal kirliliğini belirlemek, toprak ve bitkideki ağır metal miktarının karayoluna olan uzaklığı ile olası değişimini ortaya koymak amaçlanmıştır. Karayolunun trafik yoğunluğu dikkate alınarak 8 farklı örnekleme noktası belirlenmiştir. Karayolunun belirlenen her bir örnekleme noktasının sağ ve sol tarafindan yola $25,100,500 \mathrm{~m}$ ve $1 \mathrm{~km}$ uzaklıktan, 0-30 cm derinlikten toprak $(\mathrm{N}=64)$ ve alınan topraklarda yetiştirilen bitki $(\mathrm{N}=12)$ örnekleri alınmıştır. Alınan topraklardaki ağır metaller dietilen triamin pentaasetik asit (DTPA), bitkilerde ise yaş yakma yöntemiyle ekstrakte edilmiştir ve ölçümler indüktif olarak eşleşmiş plazma optik emisyon spektrometresi (ICP-OES) cihazı kullanılarak yapılmıştır.

Çalışma sonucunda toprak örneklerinde; $\mathrm{Pb} 0,130-0,780 \mathrm{mg} / \mathrm{kg}, \mathrm{Cd} 0-0,265 \mathrm{mg} / \mathrm{kg}, \mathrm{Ni}$ 0,370-3,97 mg/kg, Cr 0-0,120 mg/kg, Co 0-1,83 mg/kg, Al 0-0,700 mg/kg, Fe 1,45-22,8 mg/kg, Cu 0,385-5,43 mg/kg, Mn 1,96-27 mg/kg Zn 0-4,26 mg/kg ve bitki örneklerinde $\mathrm{Pb} 0-0,155 \mathrm{mg} / \mathrm{kg}$, Cd 0-0,105 mg/kg, Ni 0,100$3,53 \mathrm{mg} / \mathrm{kg}$, Cr 0,0 mg/kg, Co 0,0 mg/kg, Al 0,0 mg/kg, Fe 5,60-25,0 mg/kg, Cu 0,121-4,48 mg/kg, Mn $2,35-15,4 \mathrm{mg} / \mathrm{kg}, \mathrm{Zn} 0,554-6,75 \mathrm{mg} / \mathrm{kg}$ aralığında bulunmuştur.

Yapılan bu çalışma sonucunda elde edilen toprak numunelerine ait veriler Türkiye ve Dünyada toprakta müsaade edilen ağır metal sınır değerleri ile karşılaştırıldığında; toprakta ağır metal birikiminin kabul edilebilir sınır değeri aşmadığı görülmüştür. Bitki örneklerine ait veriler incelendiğinde ağır metal birikiminin olduğu tespit edilmiştir, ancak Dünya Sağlık Örgütünün (WHO) ve Birleşmiş Milletler Gıda ve Tarım Örgütünün (FAO) bitkilerde müsaade ettiği ağır metal sınır değerleri ile karşılaştırıldığında ağır metal birikiminin kabul edilebilir sınır değeri aşmamış olduğu görülmüştür. Ayrıca mesafeye bağlı olarak karayolundan uzaklaştıkça bitki ve topraktaki ağır metal seviyelerinin azaldığı tespit edilmiştir. Bu durum toprakta ve de bitkide mevcut olan ağır metallerin karayolu kaynaklı olabileceğini göstermiştir.

Anahtar Kelimeler: Toprak kirliliği, Ağır metal, DTPA yöntemi, ICP-OES

\footnotetext{
*Sorumlu yazar (Corresponding author): Abdulah ÖZKAN, abdullah.ozkan@iste.edu.tr
} 


\title{
Heavy Metal Pollution in Agricultural Lands and Plants around Antakya- Cilvegözü Highway
}

\begin{abstract}
The purpose of this study is to determine heavy metal pollution of the agricultural lands and the plants growing in these lands around Antakya-Cilvegözü international highway in Hatay province based on traffic and agricultural activities and also to reveal possible changes of the heavy metal amount in soil and plants with distance to highway. 8 different sampling points are determined considering traffic intensity of the road. Soil $(\mathrm{N}=64)$ and plant $(\mathrm{N}=12)$ samples are taken from 0-30 cm depth and 25, $100,500 \mathrm{~m}$ away from both right and left side of the particular sampling points of the highway. Heavy metals in the soil are extracted with diethylenetriaminepentaacetic acid (DTPA) while wet burning method is applied for heavy metal extraction from plants. Measurements are made with Inductively Coupled Plasma Optical Emission Spectrometry (ICP-OES) instrument.

According to the analyses, the result of soils $\mathrm{Pb} 0.130-0.780 \mathrm{mg} / \mathrm{kg}, \mathrm{Cd} 0-0.265 \mathrm{mg} / \mathrm{kg}, \mathrm{Ni} 0.370-3.97$ $\mathrm{mg} / \mathrm{kg}$, Cr 0-0.120 mg/kg, Co 0-1.83 mg/kg, Al 0-0.700 mg/kg, Fe 1.45-22.8 mg/kg, Cu 0.385-5.43 $\mathrm{mg} / \mathrm{kg}, \mathrm{Mn} 1.96-27 \mathrm{mg} / \mathrm{kg} \mathrm{Zn} \mathrm{0-4.26} \mathrm{mg/kg} \mathrm{and} \mathrm{the} \mathrm{result} \mathrm{of} \mathrm{plants} \mathrm{Pb} 0-0.155 \mathrm{mg} / \mathrm{kg}, \mathrm{Cd} \mathrm{0-0.105} \mathrm{mg/kg,}$ Ni $0.100-3.53 \mathrm{mg} / \mathrm{kg}$, Cr $0.0 \mathrm{mg} / \mathrm{kg}$, Co $0.0 \mathrm{mg} / \mathrm{kg}$, Al $0.0 \mathrm{mg} / \mathrm{kg}$, Fe 5.60-25.0 mg/kg, Cu 0.121-4.48 $\mathrm{mg} / \mathrm{kg}, \mathrm{Mn} 2.35-15.4 \mathrm{mg} / \mathrm{kg}, \mathrm{Zn} 0.554-6.75 \mathrm{mg} / \mathrm{kg}$ were found.

Results of the study shows that datas obtained from soil samples are within the permissible range compared to heavy metal limitations both in Turkey and the World. Heavy metal accumulation is observed in plant samples. However, compared to World Health Organization (WHO) and United Nations Food and Agriculture Organization (FAO) limitations, it is seen that heavy metal accumulation is within the permissible range. Additionally, it is revealed that heavy metal concentration in soil and plants is decreasing depending on the distance away from highway. This shows that the heavy metal present in soil and plants should be originating from highway.
\end{abstract}

Keywords: Soil pollution, Heavy metal, DTPA method, ICP-OES

\section{GİRIŞ}

Tarım toprakları, ülkemizin ekonomik yönden gelişmesini ve insanlarımızın refahının sürekliliğini sağlayacak en önemli doğal kaynaklarımızdan birisidir. Tarım topraklarının bu önemi, üretilemeyen ve yenilenmesi nerdeyse mümkün olmayan tek kaynak olmasından ileri gelmektedir. Uzunca bir zamandan beri ülkemizde tarıma açılan yeni alan olmadığı göz önünde bulundurulduğunda ülkemizin tarıma açılacak yeni alanlarının artık tükenme noktasına geldiğini göstermektedir. Ayrıca nüfus artışı ve gelişen teknolojiye bağlı olarak artan insan ihtiyaçlarının karşılanabilmesi için yoğun tarımsal faaliyetler yapilmaktadir. Bunun sonucunda tarım alanlarımızda kirlenme tehdidi başlamış ve toprak kalitesinin her geçen gün daha da kötüye gittiği tespit edilmiştir.

Toprağın üzerindeki tarımsal ve endüstriyel faaliyetler sonucunda doğal yapısı tamamen veya kısmen yabancı maddeler tarafindan kirletilir. $\mathrm{Bu}$ maddelerin pek çoğu toprağın bünyesinde belirli oranlarda ve çoğunlukla eser miktarlarda bulunur. $\mathrm{Bu}$ bakımdan kimyasal kirlilik denince ilk akla gelen kirleticiler ağır metallerdir [1]. Motorlu taşıtlar ve bilinçsizce yapılan tarım faaliyetleri ağır metal kirliliğinin önemli bir bölümünden sorumludur [2]. 
Ağır metaller yoğunluğu $5 \mathrm{~g} / \mathrm{cm}^{3}$, den büyük olan metallerdir. Bu grupta kurşun, kadmiyum, krom, demir, kobalt, bakır, nikel, civa ve çinko dâhil olmak üzere 60'tan fazla metal yer almaktadır [3]. Motorlu taşı trafiğinden kaynaklanan ağır metallere bakıldığında; kurşun (Pb), nikel (Ni), civa $(\mathrm{Hg})$, kadmiyum $(\mathrm{Cd})$, krom $(\mathrm{Cr})$, demir $(\mathrm{Fe})$, bakır $(\mathrm{Cu})$, mangan $(\mathrm{Mn})$ ve çinko $(\mathrm{Zn})$ görülürken, tarımsal faaliyetlerden kaynaklı ağır metaller ise; Kadmiyum (Cd), krom (Cr), bakır (Cu) ve çinko $(\mathrm{Zn})$ 'dur.

Kurşun $(\mathrm{Pb})$ benzine katılan Tetra Etil Kurşun (TEK)'dan, nikel (Ni) dizel yakıtından ve motor yağlarından kirletici olarak açığa çıkmaktadır. Kadmiyum (Cd) ve çinko ( $\mathrm{Zn}$ ) ise araç lastikleri, yağlar ve diğer araç donanımları ve fosforlu gübrelerden kaynaklanmaktadır [4,5]. Bakır, demir, mangan ve krom kirliliğinin ise motorlu taşıtların yanı sıra çeşitli tarımsal faaliyetlerden kaynaklandığı bilinmektedir [6].

Karayolundaki yoğun araç trafiği ve çevresinde gerçekleştirilen tarımsal faaliyetler, özellikle tarımsal ürünlerle insan sağlığını tehdit edecek sorunları beraberinde getirmektedir [6]. Bu yüzden ağır metallerin toplam miktarlarının belirlenmesi oldukça büyük öneme sahiptir. Hatta bitkiler tarafından alınabilir formda bulunan ağır metal miktarlarının ölçülmesi çevre kirliliği ve metallerin toksik etkilerinin tespiti açısından çok daha fazla önem arz etmektedir.

$\mathrm{Bu}$ çalışmayla, Hatay İli sınırları içerisinde yer alan Antakya-Cilvegözü uluslararası karayolu etrafındaki topraklar ve bu topraklarda yetişen bitkilerdeki ağır metal seviyesi incelenmiştir. Trafik ve tarımsal faaliyet kaynaklı ağır metal kirliliği belirlenmiş ve karayoluna uzaklığı ile olası değişimi ortaya konulmuştur.

\section{2. ÖNCEKİ ÇALIŞMALAR}

Özkan ve arkadaşları [7], Hatay havaalanı etrafındaki tarım arazilerinin ağır metal kirliliğini tespit etmek için 27 adet toprak örneğini incelemiştir. Yapılan çalışma sonucunda herhangi bir ağır metal kirliliğine rastlanılmamıştır. Ancak havaalanından uzaklaştıkça söz konusu ağır metal miktarlarının azaldığını tespit etmişler. Dolayısıyla toprakta mevcut olan ağır metallerin sebebinin havayolu trafiği kaynaklı olabileceğini ifade etmişler. Karaçağıl [8], İstanbul'un Anadolu yakası sahil şeridi yeşil alanlarının 202 farklı noktasından 404 adet toprak örneği alarak ağır metal kirliliğini tespit etmeye çalışmıştır. Aynı zamanda bu çalışmada $\mathrm{pH}$, organik madde, kireç ve fosfor analizlerini de yapmıştır. Çalışma sonucunda 7 adet örnekte krom, 2 adet örnekte nikel ve 1 adet örnekte bakır miktarlarının yüksek, diğer örneklerin ise normal sınırlar içerinde olduğunu tespit etmiştir.

Knezeviç ve arkadaşları [9], Polonya'nın farklı bölgelerinden almış oldukları toprak ve bitki örneklerini inceleyerek olası ağır metal kirliliğini tespit etmeye çalışmıştır. Çalışma sonucunda karayoluna yakın bölgelerden alınan örneklerdeki

$\mathrm{Pb}, \mathrm{Ni}, \mathrm{Zn}$ ve Mn gibi ağır metal miktarlarının, karayoluna nispeten daha uzak bölgelerden alınan örneklerdekinden daha yüksek olduğunu tespit etmişlerdir. Ayrıca Tao ve arkadaşları [10], Çin'in Shanghai ağır metal kirliliğini belirlemek için toprak örnekleri alarak $\mathrm{Pb}, \mathrm{Zn}, \mathrm{Cu}, \mathrm{Cr}, \mathrm{Cd}$ ve $\mathrm{Ni}$ analizleri yapmış ve sonuçların oldukça yüksek seviyede olduğunu tespit etmişlerdir. Yapmış oldukları çalışmayla topraklardaki ağır metal kirliğinin nedeninin trafik ve sanayi olduğunu belirtmişlerdir. Möller ve arkadaşları [11], Damascus Ghouta şehrinde trafiğin yoğun olduğu 22 farklı bölgeden 51 adet toprak örneği alarak $\mathrm{Pb}$, $\mathrm{Cu}$ ve $\mathrm{Zn}$ analizleri yapmış ve bahsi geçen elementlerin miktarlarının çok yüksek olduğunu tespit etmişlerdir. Bunun yanı sıra Sezgin ve arkadaşları [12], İstanbul'un Topkapı-Avcılar güzergâhı üzerindeki E-5 karayolunun 18 km' lik bölümünden 22 farklı noktadan almış oldukları toprak örneklerinde; $\mathrm{Pb}, \mathrm{Cu}$ ve $\mathrm{Zn}$ seviyelerinin sınır değerlerinin çok üzerinde olduğunu tespit etmişlerdir. Benzer olarak Haktanır ve arkadaşları [13], Etimesgut-Ankara karayolunda $\mathrm{Pb}, \mathrm{Cd}$ ve $\mathrm{Cu}$ ağır metallerinin kirliliği araştırılmışlardır. Yol boyunca uzanan toprakların üst $0-5 \mathrm{~cm}$ toprak 
katında yüksek düzeyde $\mathrm{Pb}$ olduğunu bulmuşlardır. Ağır metal kirliliğinin yol kenarından uzaklaştıkça azaldığ 40 m'den sonra normal değerlere indiğini tespit etmişlerdir.

\section{MATERYAL VE METOT}

\section{1. Çalışma Alanı}

Türkiye'nin Ortadoğu'ya açılan en önemli uluslararası taşımacılık yolu olan AntakyaCilvegözü uluslararası karayolu Hatay İli sınırları içerisinde yer almaktadır. 48,5 km uzunluğunda, rakımı (deniz seviyesinden yüksekliği) 301 metre, $36^{\circ} 14^{\prime} 8,9808^{\prime \prime}$ ve $36^{\circ} 39^{\prime} 47,6856^{\prime \prime}$ GPS koordinatlarına sahiptir.

\section{2. Örnek Toplama}

Antakya-Cilvegözü karayolunun belirlenen 8 farklı noktasının sağ ve sol tarafindan yola 25 , $100,500 \mathrm{~m}$ ve $1 \mathrm{~km}$ uzaklıkta olacak şekilde, 0-30 cm derinlikten toplam 64 toprak ve 4 pamuk, 4 misır ve 4 ayçiçeği bitkisi örneği alınmış ve plastik poşetlere konularak laboratuvara getirilmiştir.

\section{3. Örneklerin Hazırlanması}

Toprak örnekleri, açık havada kurutulduktan sonra, havanda dövülerek homojen hale getirilmiştir. Daha sonra analiz türüne uygun çaptaki paslanmaz çelik elekten geçirilmiştir.

Bitki örnekleri ise; iyice temizlendikten sonra etüv kullanılarak kurutulmuştur. Ardından ögütülerek homojenize edilmiştir [14].

\subsection{Metot}

Toprak örneklerindeki ağır metaller, DTPA çözeltisi [(1:2); (G:V)] ile ekstrakte edilmiş ve ICP-OES cihazı kullanılarak analiz edilmiştir [15]. Bitki örneklerindeki ağır metaller; nitrik asit: hidrojen peroksit $\left(\mathrm{HNO}_{3}: \mathrm{H}_{2} \mathrm{O}\right)$ karışımı [(8:2) $(\mathrm{V}: \mathrm{V})]$ kullanılarak mikrodalga firında yakılmış ve ICP-OES cihazıyla analiz edilmiştir.
Ayrıca toprak örneklerinde; kalsimetre yöntemiyle volümetrik olarak \% kireç [16], modifiye edilmiş Walkley-Black metoduyla \% organik madde [17], 1:2,5 (w/v) toprak: su süspansiyonunda kombine elektrotlu $\mathrm{pH}$ metre ile $\mathrm{pH}$ [18] ve Bouyoucos Hidrometre yöntemi ile bünye analizleri [19] gibi rutin fizikokimyasal analizler de yapılmıştır.

\subsection{ICP-OES Optimizasyonu}

Ağır metal analizleri ICP-OES cihazıyla yapılmıştır. Her bir ağır metal elementine ait korelasyon katsayısı $\left(\mathrm{R}^{2}\right)$, dedeksiyon limiti (LOD) ve tayin sınırı (LOQ) değerleri Çizelge 1'de verilmiştir.

Çizelge 1. Ağır metallerin $\mathrm{R}^{2}$, LOD ve LOQ değerleri

\begin{tabular}{|c|c|c|c|}
\hline Ağır Metal & $\begin{array}{c}\text { Korelasyon } \\
\text { Katsayısı }\left(R^{2}\right)\end{array}$ & $\begin{array}{c}\text { LOD } \\
(\mathrm{mg} / \mathrm{kg})\end{array}$ & $\begin{array}{c}\text { LOQ } \\
(\mathrm{mg} / \mathrm{kg})\end{array}$ \\
\hline $\mathrm{Fe}$ & 0,999 & 0,343 & 1,142 \\
\hline $\mathrm{Zn}$ & 0,996 & 0,705 & 2,348 \\
\hline $\mathrm{Cd}$ & 0,997 & 0,115 & 0,382 \\
\hline $\mathrm{Cu}$ & 0,999 & 0,256 & 0,852 \\
\hline $\mathrm{Co}$ & 0,999 & 0,202 & 0,673 \\
\hline $\mathrm{Ni}$ & 0,999 & 0,118 & 0,393 \\
\hline $\mathrm{Al}$ & 0,999 & 0,013 & 0,041 \\
\hline $\mathrm{Mn}$ & 0,999 & 0,059 & 0,196 \\
\hline $\mathrm{Pb}$ & 0,999 & 0,088 & 0,293 \\
\hline $\mathrm{Cr}$ & 0,999 & 0,010 & 0,034 \\
\hline
\end{tabular}

\section{ARAŞTIRMA BULGULARI}

Avrupa ülkeleri ağır metal sınır değerleri Çizelge 2, Türkiye Çevre ve Orman Genel Müdürlüğünün toprakta müsaade edilen ağır metal sınır değerleri Çizelge 3 ve WHO/FAO'nun bitkilerde müsaade ettiği ağır metal sınır değerleri Çizelge 4'te verilmiştir. 
Çizelge 2. Avrupa ülkeleri ağır metal sınır değerleri (mg/kg) [20]

\begin{tabular}{|c|c|c|c|c|c|c|c|}
\hline Ülke & $\begin{array}{l}\text { Ülke Kalite } \\
\text { Std. }\end{array}$ & $\mathrm{Cd}$ & $\mathrm{Cr}$ & $\mathrm{Cu}$ & $\mathrm{Ni}$ & $\mathrm{Pb}$ & $\mathrm{Zn}$ \\
\hline Belçika & $\begin{array}{c}\text { Tarım } \\
\text { Bak.'lığ } 1\end{array}$ & 1,5 & 70 & 90 & 20 & 120 & 300 \\
\hline Almanya & $\begin{array}{c}\text { Biyoatık } \\
\text { Yönet. Tip II }\end{array}$ & 1,5 & 100 & 100 & 50 & 150 & 400 \\
\hline İ́rlanda & Taslak & 1,5 & 100 & 100 & 50 & 150 & 350 \\
\hline Hollanda & $\begin{array}{c}\text { İkinci Sınıf } \\
\text { Kompost }\end{array}$ & 1 & 50 & 60 & 20 & 100 & 200 \\
\hline İ́spanya & A sinıfi & 2 & 100 & 100 & 60 & 150 & 400 \\
\hline İsveç & $\begin{array}{c}\text { Kalite } \\
\text { Güvence Org. }\end{array}$ & 1 & 100 & 100 & 50 & 100 & 300 \\
\hline İngiltere & $\begin{array}{c}\text { TCA Kalite } \\
\text { Etiketi }\end{array}$ & 1,5 & 100 & 200 & 50 & 150 & 400 \\
\hline
\end{tabular}

Çizelge 3. Türkiye ağır metal sınır değerleri $(\mathrm{mg} / \mathrm{kg})[21]$.

\begin{tabular}{|c|c|c|}
\hline $\begin{array}{c}\text { Ağır } \\
\text { Metal }\end{array}$ & $\begin{array}{c}\text { pH 5-6 } \\
(\mathrm{mg} / \mathrm{kg} \mathrm{kuru} \text { toprak })\end{array}$ & $\begin{array}{c}\mathrm{pH}>6 \\
(\mathrm{mg} / \mathrm{kg} \mathrm{kuru} \text { toprak })\end{array}$ \\
\hline $\mathrm{Pb}$ & 50 & 300 \\
\hline $\mathrm{Cd}$ & 1 & 3 \\
\hline $\mathrm{Ni}$ & 30 & 75 \\
\hline $\mathrm{Cr}$ & 100 & 100 \\
\hline $\mathrm{Co}$ & 80 & 80 \\
\hline $\mathrm{Cu}$ & 50 & 140 \\
\hline $\mathrm{Fe}$ & 4,5 & 4,5 \\
\hline $\mathrm{Zn}$ & 150 & 300 \\
\hline $\mathrm{Mn}$ & 70 & 70 \\
\hline
\end{tabular}

Çizelge 4. FAO/WHO'nun bitkilerde kabul ettiği ağır metal sınır değerleri (mg/kg) [22]

\begin{tabular}{|c|c|}
\hline Ă̆ır Metal & Sinır Değer $(\mathrm{mg} / \mathrm{kg})$ \\
\hline $\mathrm{Pb}$ & 2 \\
\hline $\mathrm{Cd}$ & 0,5 \\
\hline $\mathrm{Ni}$ & 5 \\
\hline $\mathrm{Cr}$ & 0,5 \\
\hline $\mathrm{Cu}$ & 5 \\
\hline $\mathrm{Fe}$ & 30 \\
\hline $\mathrm{Zn}$ & 50 \\
\hline
\end{tabular}

\subsection{Toprak Örneklerinin Fiziksel ve Kimyasal Özellikleri}

Toplanan toprak örneklerinin fiziksel ve kimyasal analizlerinin sonuçlarına göre; $\mathrm{pH}$ 7,5 ile 8,6 arasında; kireç \%15,0 ile \%35,8 arasında ve organik madde $\% 1,50$ ile $\% 4,31$ arasında değişmektedir. Araştırmada kullanılan toprak örneklerinin çoğunluğu nötr ve hafif alkalin reaksiyonlu, büyük bir bölümü ise az kireçlidir.
Organik madde içerikleri genellikle yetersizdir. Toprak örneklerinin tekstürleri Kumlu Killi Tin'dan, Kumlu Kil'e kadar geniş bir varyasyona sahiptir.

\subsection{Toprak Örneklerinin Ăğır Metal İçerikleri}

Analizi yapılan toprak ve bitki örneklerinin ağır metal içerikleri Çizelge 5 (a ve b) ve 6'da (standart sapmalarıyla) verilmiştir.

\section{SONUÇ ve ÖNERILER}

Çalışma sonucunda; toprak örneklerinin ağır metal içerikleri

$\mathrm{Pb} \quad 0,130-0,780 \mathrm{mg} / \mathrm{kg}, \quad \mathrm{Cd} \quad 0-0,265 \mathrm{mg} / \mathrm{kg}$, $\mathrm{Ni} \quad 0,370-3,97 \mathrm{mg} / \mathrm{kg}, \quad \mathrm{Cr} \quad 0-0,120 \quad \mathrm{mg} / \mathrm{kg}$, Co $\quad 0-1,83 \quad \mathrm{mg} / \mathrm{kg}, \quad$ Al $\quad 0-0,700 \quad \mathrm{mg} / \mathrm{kg}$, $\mathrm{Fe} \quad 1,45-22,8 \mathrm{mg} / \mathrm{kg}, \quad \mathrm{Cu} \quad 0,385-5,43 \mathrm{mg} / \mathrm{kg}$, Mn 1,96-27 mg/kg ve $\mathrm{Zn} \mathrm{0-4,26} \mathrm{mg/kg,} \mathrm{bitki}$ örneklerinin ağı metal içerikleri de $\mathrm{Pb} \quad 0-0,155 \quad \mathrm{mg} / \mathrm{kg}, \quad \mathrm{Cd} \quad 0-0,105 \quad \mathrm{mg} / \mathrm{kg}$, Ni 0,100-3,53 mg/kg, Cr 0,0 mg/kg, Co 0,0 mg/kg, Al $\quad 0,0 \quad \mathrm{mg} / \mathrm{kg}, \quad$ Fe $5,60-25,0 \quad \mathrm{mg} / \mathrm{kg}$, $\mathrm{Cu}$ 0,121-4,48 mg/kg, Mn 2,35-15,4 mg/kg, Zn 0,554-6,75 mg/kg olarak bulunmuştur.

Analizi yapılan toprak ve bitki örneklerinin ağır metal değerleri, müsaade edilen ağır metal sınır değerleri (Çizelge 2, 3 ve 4) ile karşılaştırıldığında genel olarak normal sinırlar içerisinde olduğu tespit edilmiştir. Sadece topraktaki Fe elementinin sınır değerlerin üzerinde olduğu görülmektedir. Ayrıca Fe elementi dişındaki diğer tüm elementlerin karayolundan uzaklaştıkça miktarlarında düşüş olduğu gözlemlenmiştir.

Fakat bitki örneklerinde; $\mathrm{Fe}, \mathrm{Cu}, \mathrm{Mn}$ ve $\mathrm{Zn}$ değerlerinin rastgele değişkenlik gösterdiği, $\mathrm{Pb}, \mathrm{Cd}$ ve Ni değerlerinin tıpkı toprak örneklerinde olduğu gibi karayolundan uzaklaştıkça azaldığı, $\mathrm{Cr}$, Co ve Al'un ise hiç olmadığı tespit edilmiştir.

Yapılan bu çalışma topraktaki Fe, bitkideki Fe, $\mathrm{Cu}$, $\mathrm{Mn}$ ve $\mathrm{Zn}$ varlığının tarımsal faaliyetler ve geriye kalan $\mathrm{Pb}, \mathrm{Cd}$, Ni gibi ağır metallerin var olma sebebinin karayolu trafiği olduğunu ortaya koymuştur. 
Antakya-Cilvegözü Karayolu Etrafindaki Tarım Arazilerinde ve Bitkilerdeki Ă̆ır Metal Kirliliği

Çizelge 5.a. Toprak örneklerinin ağır metal analiz sonuçları (mg/kg)

\begin{tabular}{|c|c|c|c|c|c|c|c|c|c|c|c|}
\hline $\begin{array}{c}\text { Örnek } \\
\text { Bölgesi }\end{array}$ & $\begin{array}{c}\text { Karayoluna } \\
\text { Uzaklık }\end{array}$ & $\mathbf{P b}$ & Cd & $\mathbf{N i}$ & $\mathrm{Cr}$ & Co & Al & $\mathbf{F e}$ & $\mathrm{Cu}$ & Mn & Zn \\
\hline \multirow{4}{*}{$\begin{array}{c}\text { Bölge 1 } \\
\text { (Karayolu } \\
\text { sağ tarafi) }\end{array}$} & $25 \mathrm{~m}$ & $0,770 \pm 0,04$ & $0,265 \pm 0,04$ & $3,57 \pm 0,13$ & $0,095 \pm 0,05$ & $1,83 \pm 0,08$ & $0,700 \pm 0,05$ & $16,2 \pm 1,18$ & $5,23 \pm 0,12$ & $27,0 \pm 0,34$ & $4,26 \pm 0,15$ \\
\hline & $100 \mathrm{~m}$ & $0,740 \pm 0,01$ & $0,250 \pm 0,03$ & $3,50 \pm 0,15$ & - & $1,76 \pm 0,11$ & $0,652 \pm 0,06$ & $17,3 \pm 0,48$ & $5,20 \pm 0,21$ & $25,2 \pm 0,77$ & $4,03 \pm 0,11$ \\
\hline & $500 \mathrm{~m}$ & $0,550 \pm 0,01$ & $0,221 \pm 0,01$ & $3,01 \pm 0,16$ & - & $1,65 \pm 0,13$ & $0,641 \pm 0,07$ & $15,3 \pm 1,10$ & $3,46 \pm 0,16$ & $24,9 \pm 0,34$ & $3,95 \pm 0,08$ \\
\hline & $1 \mathrm{~km}$ & $0,480 \pm 0,03$ & $0,192 \pm 0,05$ & $3,05 \pm 0,11$ & - & $1,60 \pm 0,11$ & $0,603 \pm 0,11$ & $15,6 \pm 0,19$ & $4,05 \pm 0,19$ & $23,5 \pm 0,17$ & $4,01 \pm 0,13$ \\
\hline \multirow{4}{*}{$\begin{array}{c}\text { Bölge 1 } \\
\text { (Karayolu } \\
\text { sol tarafi) }\end{array}$} & $25 \mathrm{~m}$ & $0,780 \pm 0,06$ & $0,260 \pm 0,03$ & $3,97 \pm 0,11$ & $0,100 \pm 0,05$ & $1,75 \pm 0,09$ & $0,700 \pm 0,06$ & $16,0 \pm 0,50$ & $5,43 \pm 0,31$ & $27,0 \pm 0,51$ & $4,26 \pm 0,16$ \\
\hline & $100 \mathrm{~m}$ & $0,750 \pm 0,01$ & $0,240 \pm 0,05$ & $3,65 \pm 0,09$ & - & $1,71 \pm 0,13$ & $0,695 \pm 0,05$ & $18,5 \pm 0,38$ & $5,10 \pm 0,18$ & $25,2 \pm 0,64$ & $4,11 \pm 0,06$ \\
\hline & $500 \mathrm{~m}$ & $0,630 \pm 0,03$ & $0,180 \pm 0,02$ & $3,60 \pm 0,16$ & - & $1,65 \pm 0,15$ & $0,690 \pm 0,08$ & $15,1 \pm 1,12$ & $5,00 \pm 0,11$ & $24,9 \pm 0,71$ & $4,05 \pm 0,05$ \\
\hline & $1 \mathrm{~km}$ & $0,550 \pm 0,02$ & $0,180 \pm 0,03$ & $3,59 \pm 0,13$ & - & $1,58 \pm 0,16$ & $0,700 \pm 0,08$ & $13,6 \pm 0,35$ & $4,45 \pm 0,21$ & $23,0 \pm 0,65$ & $3,96 \pm 0,08$ \\
\hline \multirow{4}{*}{$\begin{array}{c}\text { Bölge } 2 \\
\text { (Karayolu } \\
\text { sağ tarafi) }\end{array}$} & $25 \mathrm{~m}$ & $0,552 \pm 0,01$ & $0,030 \pm 0,01$ & $0,600 \pm 0,05$ & - & - & - & $5,47 \pm 0,11$ & $1,88 \pm 0,02$ & $2,87 \pm 0,18$ & $0,148 \pm 0,03$ \\
\hline & $100 \mathrm{~m}$ & $0,550 \pm 0,03$ & - & $0,660 \pm 0,03$ & - & - & - & $4,34 \pm 0,17$ & $1,88 \pm 0,06$ & $2,38 \pm 0,12$ & $0,111 \pm 0,06$ \\
\hline & $500 \mathrm{~m}$ & $0,451 \pm 0,05$ & - & $0,530 \pm 0,05$ & - & - & - & $5,38 \pm 0,25$ & $1,57 \pm 0,09$ & $2,01 \pm 0,16$ & $0,101 \pm 0,05$ \\
\hline & $1 \mathrm{~km}$ & $0,490 \pm 0,03$ & - & $0,500 \pm 0,15$ & - & - & - & $1,45 \pm 0,11$ & $1,53 \pm 0,04$ & $1,96 \pm 0,21$ & $0,101 \pm 0,01$ \\
\hline \multirow{4}{*}{$\begin{array}{c}\text { Bölge } 2 \\
\text { (Karayolu } \\
\text { sol tarafi) }\end{array}$} & $25 \mathrm{~m}$ & $0,581 \pm 0,01$ & $0,220 \pm 0,01$ & $1,30 \pm 0,16$ & - & $0,780 \pm 0,08$ & $0,450 \pm 0,09$ & $9,15 \pm 0,16$ & $3,14 \pm 0,21$ & $16,1 \pm 0,13$ & $3,01 \pm 0,07$ \\
\hline & $100 \mathrm{~m}$ & $0,551 \pm 0,03$ & $0,200 \pm 0,02$ & $1,23 \pm 0,13$ & - & $0,650 \pm 0,14$ & $0,410 \pm 0,08$ & $8,71 \pm 0,25$ & $2,99 \pm 0,16$ & $16,0 \pm 0,21$ & $3,00 \pm 0,13$ \\
\hline & $500 \mathrm{~m}$ & $0,500 \pm 0,02$ & $0,175 \pm 0,05$ & $1,15 \pm 0,13$ & - & $0,640 \pm 0,09$ & $0,350 \pm 0,01$ & $10,1 \pm 0,13$ & $3,05 \pm 0,21$ & $15,5 \pm 0,16$ & $2,91 \pm 0,11$ \\
\hline & $1 \mathrm{~km}$ & $0,450 \pm 0,01$ & $0,150 \pm 0,03$ & $1,00 \pm 0,10$ & - & $0,650 \pm 0,13$ & $0,305 \pm 0,15$ & $6,55 \pm 0,35$ & $2,85 \pm 0,19$ & $14,5 \pm 0,09$ & $2,85 \pm 0,16$ \\
\hline \multirow{4}{*}{$\begin{array}{c}\text { Bölge } 3 \\
\text { (Karayolu } \\
\text { sağ tarafi) }\end{array}$} & $25 \mathrm{~m}$ & $0,450 \pm 0,01$ & $0,155 \pm 0,04$ & $0,511 \pm 0,06$ & $0,010 \pm 0,005$ & $0,25 \pm 0,08$ & - & $6,32 \pm 0,18$ & $2,01 \pm 0,16$ & $8,58 \pm 0,16$ & - \\
\hline & $100 \mathrm{~m}$ & $0,510 \pm 0,01$ & $0,150 \pm 0,06$ & $0,505 \pm 0,05$ & - & $0,21 \pm 0,05$ & - & $3,83 \pm 0,08$ & $1,50 \pm 0,15$ & $7,92 \pm 0,27$ & - \\
\hline & $500 \mathrm{~m}$ & $0,420 \pm 0,04$ & $0,121 \pm 0,01$ & $0,414 \pm 0,07$ & - & - & - & $7,24 \pm 0,16$ & $1,26 \pm 0,21$ & $7,89 \pm 0,14$ & - \\
\hline & $1 \mathrm{~km}$ & $0,380 \pm 0,03$ & $0,112 \pm 0,03$ & $0,370 \pm 0,11$ & - & - & - & $5,61 \pm 0,19$ & $1,25 \pm 0,19$ & $7,51 \pm 0,17$ & - \\
\hline \multirow{4}{*}{$\begin{array}{c}\text { Bölge } 3 \\
\text { (Karayolu } \\
\text { sol tarafi) }\end{array}$} & $25 \mathrm{~m}$ & $0,562 \pm 0,04$ & $0,100 \pm 0,03$ & $0,630 \pm 0,16$ & - & - & - & $3,62 \pm 0,15$ & $1,93 \pm 0,04$ & $6,10 \pm 0,17$ & $0,190 \pm 0,05$ \\
\hline & $100 \mathrm{~m}$ & $0,560 \pm 0,02$ & $0,080 \pm 0,01$ & $0,600 \pm 0,06$ & - & - & - & $3,43 \pm 0,25$ & $1,86 \pm 0,03$ & $4,51 \pm 0,13$ & $0,160 \pm 0,03$ \\
\hline & $500 \mathrm{~m}$ & $0,551 \pm 0,06$ & - & $0,560 \pm 0,05$ & $0,120 \pm 0,01$ & - & - & $4,09 \pm 0,16$ & $1,61 \pm 0,07$ & $4,05 \pm 0,21$ & $0,160 \pm 0,03$ \\
\hline & $1 \mathrm{~km}$ & $0,510 \pm 0,03$ & - & $0,510 \pm 0,13$ & $0,116 \pm 0,05$ & - & - & $3,29 \pm 0,21$ & $1,56 \pm 0,05$ & $3,86 \pm 0,18$ & $0,110 \pm 0,01$ \\
\hline \multirow{4}{*}{$\begin{array}{c}\text { Bölge } 4 \\
\text { (Karayolu } \\
\text { sağ tarafi) }\end{array}$} & $25 \mathrm{~m}$ & $0,182 \pm 0,03$ & $0,085 \pm 0,01$ & $1,23 \pm 0,15$ & - & - & $0,080 \pm 0,03$ & $5,47 \pm 0,21$ & $1,87 \pm 0,09$ & $13,5 \pm 0,10$ & - \\
\hline & $100 \mathrm{~m}$ & $0,150 \pm 0,05$ & $0,055 \pm 0,03$ & $1,21 \pm 0,10$ & - & - & $0,065 \pm 0,02$ & $3,34 \pm 0,18$ & $1,81 \pm 0,11$ & $13,4 \pm 0,12$ & - \\
\hline & $500 \mathrm{~m}$ & $0,131 \pm 0,01$ & $0,065 \pm 0,01$ & $1,15 \pm 0,05$ & - & - & $0,060 \pm 0,01$ & $5,68 \pm 0,25$ & $1,65 \pm 0,08$ & $13,1 \pm 0,16$ & - \\
\hline & $1 \mathrm{~km}$ & $0,130 \pm 0,04$ & $0,035 \pm 0,01$ & $1,13 \pm 0,05$ & - & - & $0,051 \pm 0,01$ & $4,47 \pm 0,21$ & $1,50 \pm 0,13$ & $12,8 \pm 0,21$ & - \\
\hline \multirow{4}{*}{$\begin{array}{c}\text { Bölge } 4 \\
\text { (Karayolu } \\
\text { sol tarafi) }\end{array}$} & $25 \mathrm{~m}$ & $0,411 \pm 0,03$ & - & $1,21 \pm 0,15$ & - & - & - & $5,03 \pm 0,10$ & $1,15 \pm 0,03$ & $13,8 \pm 0,13$ & $0,750 \pm 0,06$ \\
\hline & $100 \mathrm{~m}$ & $0,400 \pm 0,01$ & - & $1,15 \pm 0,03$ & - & - & - & $3,96 \pm 0,29$ & $1,10 \pm 0,05$ & $14,4 \pm 0,16$ & $0,510 \pm 0,01$ \\
\hline & $500 \mathrm{~m}$ & $0,300 \pm 0,02$ & - & $1,14 \pm 0,01$ & - & - & - & $4,42 \pm 0,36$ & $1,03 \pm 0,04$ & $12,1 \pm 0,11$ & $0,440 \pm 0,03$ \\
\hline & $1 \mathrm{~km}$ & $0,260 \pm 0,01$ & - & $1,05 \pm 0,06$ & - & - & - & $4,56 \pm 0,26$ & $1,01 \pm 0,05$ & $13,1 \pm 0,09$ & $0,415 \pm 0,05$ \\
\hline
\end{tabular}


Çizelge 5.b. Toprak örneklerinin ağır metal analiz sonuçları $(\mathrm{mg} / \mathrm{kg})$

\begin{tabular}{|c|c|c|c|c|c|c|c|c|c|c|c|}
\hline $\begin{array}{l}\text { Örnek } \\
\text { Bölgesi }\end{array}$ & $\begin{array}{c}\text { Karayoluna } \\
\text { Uzaklık }\end{array}$ & $\mathbf{P b}$ & Cd & $\mathbf{N i}$ & $\mathrm{Cr}$ & Co & Al & $\mathrm{Fe}$ & $\mathrm{Cu}$ & Mn & Zn \\
\hline \multirow{4}{*}{$\begin{array}{c}\text { Bölge } 5 \\
\text { (Karayolu } \\
\text { sağ tarafi) }\end{array}$} & $25 \mathrm{~m}$ & $0,450 \pm 0,01$ & $0,155 \pm 0,04$ & $0,511 \pm 0,06$ & $0,010 \pm 0,005$ & $0,25 \pm 0,08$ & - & $6,32 \pm 0,18$ & $2,01 \pm 0,16$ & $8,58 \pm 0,16$ & - \\
\hline & $100 \mathrm{~m}$ & $0,510 \pm 0,01$ & $0,150 \pm 0,06$ & $0,505 \pm 0,05$ & - & $0,21 \pm 0,05$ & - & $3,83 \pm 0,08$ & $1,50 \pm 0,15$ & $7,92 \pm 0,27$ & - \\
\hline & $500 \mathrm{~m}$ & $0,420 \pm 0,04$ & $0,121 \pm 0,01$ & $0,414 \pm 0,07$ & - & - & - & $7,24 \pm 0,16$ & $1,26 \pm 0,21$ & $7,89 \pm 0,14$ & - \\
\hline & $1 \mathrm{~km}$ & $0,380 \pm 0,03$ & $0,112 \pm 0,03$ & $0,370 \pm 0,11$ & - & - & - & $5,61 \pm 0,19$ & $1,25 \pm 0,19$ & $7,51 \pm 0,17$ & - \\
\hline \multirow{4}{*}{$\begin{array}{c}\text { Bölge } 5 \\
\text { (Karayolu } \\
\text { sol tarafi) }\end{array}$} & $25 \mathrm{~m}$ & $0,480 \pm 0,02$ & $0,155 \pm 0,04$ & $0,485 \pm 0,06$ & - & $0,21 \pm 0,05$ & - & $4,56 \pm 0,31$ & $2,10 \pm 0,19$ & $8,16 \pm 0,15$ & - \\
\hline & $100 \mathrm{~m}$ & $0,400 \pm 0,06$ & $0,150 \pm 0,06$ & $0,415 \pm 0,10$ & - & $0,20 \pm 0,08$ & - & $3,76 \pm 0,16$ & $1,85 \pm 0,13$ & $8,00 \pm 0,13$ & - \\
\hline & $500 \mathrm{~m}$ & $0,350 \pm 0,01$ & $0,121 \pm 0,01$ & $0,385 \pm 0,03$ & - & $0,06 \pm 0,01$ & - & $5,21 \pm 0,33$ & $1,35 \pm 0,10$ & $7,91 \pm 0,16$ & - \\
\hline & $1 \mathrm{~km}$ & $0,400 \pm 0,02$ & $0,112 \pm 0,03$ & $0,370 \pm 0,05$ & - & - & - & $4,76 \pm 0,16$ & $1,30 \pm 0,12$ & $7,65 \pm 0,08$ & - \\
\hline \multirow{4}{*}{$\begin{array}{c}\text { Bölge } 6 \\
\text { Karayolu } \\
\text { sağ tarafi) }\end{array}$} & $25 \mathrm{~m}$ & $0,261 \pm 0,03$ & - & $1,75 \pm 0,15$ & - & - & - & $15,3 \pm 0,30$ & $0,730 \pm 0,06$ & $5,65 \pm 0,10$ & - \\
\hline & $100 \mathrm{~m}$ & $0,255 \pm 0,01$ & - & $1,73 \pm 0,10$ & - & - & - & $16,2 \pm 0,26$ & $0,640 \pm 0,11$ & $5,16 \pm 0,15$ & - \\
\hline & $500 \mathrm{~m}$ & $0,232 \pm 0,03$ & - & $1,65 \pm 0,15$ & - & - & - & $16,3 \pm 0,21$ & $0,460 \pm 0,11$ & $5,16 \pm 0,11$ & - \\
\hline & $1 \mathrm{~km}$ & $0,201 \pm 0,05$ & - & $1,63 \pm 0,19$ & - & - & - & $17,6 \pm 0,43$ & $0,385 \pm 0,10$ & $5,05 \pm 0,05$ & - \\
\hline \multirow{4}{*}{$\begin{array}{c}\text { Bölge } 6 \\
\text { (Karayolu } \\
\text { sol tarafi) }\end{array}$} & $25 \mathrm{~m}$ & $0,301 \pm 0,03$ & - & $1,65 \pm 0,15$ & - & - & - & $13,6 \pm 0,61$ & $0,390 \pm 0,05$ & $6,35 \pm 0,15$ & - \\
\hline & $100 \mathrm{~m}$ & $0,250 \pm 0,06$ & - & $1,76 \pm 0,11$ & - & - & - & $13,3 \pm 0,45$ & $0,650 \pm 0,09$ & $5,15 \pm 0,21$ & - \\
\hline & $500 \mathrm{~m}$ & $0,212 \pm 0,03$ & - & $1,70 \pm 0,16$ & - & - & - & $12,5 \pm 0,36$ & $0,510 \pm 0,07$ & $5,00 \pm 0,16$ & - \\
\hline & $1 \mathrm{~km}$ & $0,181 \pm 0,04$ & - & $1,65 \pm 0,09$ & - & - & - & $13,5 \pm 0,35$ & $0,430 \pm 0,04$ & $5,00 \pm 0,11$ & - \\
\hline \multirow{4}{*}{$\begin{array}{c}\text { Bölge } 7 \\
\text { (Karayolu } \\
\text { sağ tarafi) }\end{array}$} & $25 \mathrm{~m}$ & $0,182 \pm 0,03$ & $0,085 \pm 0,01$ & $1,23 \pm 0,15$ & - & - & $0,080 \pm 0,03$ & $5,47 \pm 0,21$ & $1,87 \pm 0,09$ & $13,5 \pm 0,10$ & - \\
\hline & $100 \mathrm{~m}$ & $0,150 \pm 0,05$ & $0,055 \pm 0,03$ & $1,21 \pm 0,10$ & - & - & $0,065 \pm 0,02$ & $3,34 \pm 0,18$ & $1,81 \pm 0,11$ & $13,4 \pm 0,12$ & - \\
\hline & $500 \mathrm{~m}$ & $0,131 \pm 0,01$ & $0,065 \pm 0,01$ & $1,15 \pm 0,05$ & - & - & $0,060 \pm 0,01$ & $5,68 \pm 0,25$ & $1,65 \pm 0,08$ & $13,1 \pm 0,16$ & - \\
\hline & $1 \mathrm{~km}$ & $0,130 \pm 0,04$ & $0,035 \pm 0,01$ & $1,13 \pm 0,05$ & - & - & $0,051 \pm 0,01$ & $4,47 \pm 0,21$ & $1,50 \pm 0,13$ & $12,8 \pm 0,21$ & - \\
\hline \multirow{4}{*}{$\begin{array}{c}\text { Bölge } 7 \\
\text { (Karayolu } \\
\text { sol tarafi) }\end{array}$} & $25 \mathrm{~m}$ & $0,202 \pm 0,02$ & $0,090 \pm 0,01$ & $1,30 \pm 0,18$ & - & - & $0,110 \pm 0,03$ & $3,06 \pm 0,18$ & $1,50 \pm 0,13$ & $15,6 \pm 0,41$ & - \\
\hline & $100 \mathrm{~m}$ & $0,190 \pm 0,02$ & $0,060 \pm 0,03$ & $1,25 \pm 0,15$ & - & - & $0,100 \pm 0,03$ & $2,51 \pm 0,17$ & $1,94 \pm 0,14$ & $14,5 \pm 0,35$ & - \\
\hline & $500 \mathrm{~m}$ & $0,151 \pm 0,06$ & - & $1,05 \pm 0,13$ & - & - & $0,080 \pm 0,03$ & $2,34 \pm 0,13$ & $1,84 \pm 0,11$ & $13,2 \pm 0,91$ & - \\
\hline & $1 \mathrm{~km}$ & $0,130 \pm 0,05$ & - & $1,08 \pm 0,01$ & - & - & $0,050 \pm 0,01$ & $3,48 \pm 0,21$ & $1,61 \pm 0,08$ & $12,9 \pm 0,61$ & - \\
\hline \multirow{4}{*}{$\begin{array}{c}\text { Bölge } 8 \\
\text { (Karayolu } \\
\text { sağ tarafi) }\end{array}$} & $25 \mathrm{~m}$ & $0,751 \pm 0,05$ & $0,161 \pm 0,01$ & $2,83 \pm 0,25$ & $0,010 \pm 0,001$ & $0,681 \pm 0,07$ & $0,651 \pm 0,01$ & $22,8 \pm 0,61$ & $4,14 \pm 0,08$ & $17,3 \pm 0,11$ & $1,40 \pm 0,01$ \\
\hline & $100 \mathrm{~m}$ & $0,732 \pm 0,06$ & $0,153 \pm 0,03$ & $2,80 \pm 0,11$ & $0,010 \pm 0,005$ & $0,655 \pm 0,05$ & $0,645 \pm 0,03$ & $19,8 \pm 0,44$ & $4,10 \pm 0,05$ & $17,0 \pm 010$ & $1,35 \pm 0,03$ \\
\hline & $500 \mathrm{~m}$ & $0,685 \pm 0,03$ & $0,150 \pm 0,01$ & $2,75 \pm 0,23$ & - & $0,650 \pm 0,05$ & $0,640 \pm 0,03$ & $18,4 \pm 0,53$ & $3,95 \pm 0,03$ & $17,0 \pm 0,13$ & $1,33 \pm 0,03$ \\
\hline & $1 \mathrm{~km}$ & $0,655 \pm 0,03$ & $0,149 \pm 0,01$ & $2,75 \pm 0,13$ & - & $0,556 \pm 0,05$ & $0,640 \pm 0,01$ & $17,6 \pm 0,49$ & $3,94 \pm 0,04$ & $16,9 \pm 0,11$ & $1,25 \pm 0,01$ \\
\hline \multirow{4}{*}{$\begin{array}{c}\text { Bölge } 8 \\
\text { (Karayolu } \\
\text { sol tarafi) }\end{array}$} & $25 \mathrm{~m}$ & $0,651 \pm 0,03$ & $0,150 \pm 0,01$ & $2,83 \pm 0,25$ & - & $0,680 \pm 0,07$ & $0,680 \pm 0,05$ & $20,1 \pm 0,65$ & $4,23 \pm 0,07$ & $17,3 \pm 0,29$ & $1,35 \pm 0,06$ \\
\hline & $100 \mathrm{~m}$ & $0,640 \pm 0,06$ & $0,140 \pm 0,03$ & $2,80 \pm 0,11$ & - & $0,650 \pm 0,05$ & $0,610 \pm 0,05$ & $19,2 \pm 0,53$ & $4,01 \pm 0,21$ & $16,5 \pm 0,18$ & $1,21 \pm 0,11$ \\
\hline & $500 \mathrm{~m}$ & $0,600 \pm 0,04$ & $0,150 \pm 0,01$ & $2,75 \pm 0,23$ & - & $0,640 \pm 0,06$ & $0,510 \pm 0,08$ & $18,8 \pm 0,73$ & $3,93 \pm 0,18$ & $15,4 \pm 0,31$ & $1,20 \pm 0,05$ \\
\hline & $1 \mathrm{~km}$ & $0,555 \pm 0,05$ & $0,100 \pm 0,05$ & $2,75 \pm 0,13$ & - & $0,560 \pm 0,05$ & $0,500 \pm 0,09$ & $17,7 \pm 0,65$ & $3,85 \pm 0,16$ & $14,0 \pm 0,43$ & $1,05 \pm 0,08$ \\
\hline
\end{tabular}


Çizelge 6. Bitki örneklerinin ağır metal analiz sonuçları $(\mathrm{mg} / \mathrm{kg})$

\begin{tabular}{|c|c|c|c|c|c|c|c|c|c|c|c|}
\hline $\begin{array}{c}\text { Örnek } \\
\text { Adı }\end{array}$ & $\begin{array}{c}\text { Karayoluna } \\
\text { Uzaklık }\end{array}$ & $\mathbf{P b}$ & $\mathbf{C d}$ & $\mathbf{N i}$ & $\mathbf{C r}$ & $\mathbf{C o}$ & $\mathbf{A l}$ & $\mathbf{F e}$ & $\mathbf{C u}$ & $\mathbf{M n}$ & $\mathbf{Z n}$ \\
\hline \multirow{4}{*}{ Pamuk } & $25 \mathrm{~m}$ & $0,155 \pm 0,10$ & $0,105 \pm 0,01$ & $3,53 \pm 0,15$ & - & - & - & $25,0 \pm 0,42$ & $4,03 \pm 0,20$ & $15,35 \pm 0,20$ & $0,554 \pm 0,05$ \\
\cline { 2 - 11 } & $100 \mathrm{~m}$ & $0,155 \pm 0,08$ & $0,095 \pm 0,04$ & $2,73 \pm 0,10$ & - & - & - & $16,4 \pm 0,34$ & $3,93 \pm 0,15$ & $13,55 \pm 0,15$ & $0,680 \pm 0,07$ \\
\cline { 2 - 11 } & $500 \mathrm{~m}$ & $0,141 \pm 0,07$ & $0,065 \pm 0,01$ & $2,85 \pm 0,13$ & - & - & - & $18,2 \pm 0,35$ & $3,85 \pm 0,13$ & $15,40 \pm 0,31$ & $0,645 \pm 0,03$ \\
\cline { 2 - 11 } & $1 \mathrm{~km}$ & $0,132 \pm 0,13$ & $0,055 \pm 0,01$ & $1,77 \pm 0,05$ & - & - & - & $19,4 \pm 0,49$ & $3,77 \pm 0,15$ & $14,00 \pm 0,43$ & $0,570 \pm 0,05$ \\
\hline \multirow{4}{*}{ Misır } & $25 \mathrm{~m}$ & $0,150 \pm 0,07$ & $0,085 \pm 0,01$ & $1,75 \pm 0,15$ & - & - & - & $22,1 \pm 0,45$ & $3,53 \pm 0,15$ & $15,30 \pm 0,20$ & $6,75 \pm 0,25$ \\
\cline { 2 - 11 } & $100 \mathrm{~m}$ & $0,145 \pm 0,05$ & $0,055 \pm 0,05$ & $1,73 \pm 0,10$ & - & - & - & $17,2 \pm 0,30$ & $2,73 \pm 0,10$ & $11,35 \pm 0,15$ & $6,45 \pm 0,23$ \\
\cline { 2 - 11 } & $500 \mathrm{~m}$ & $0,131 \pm 0,04$ & $0,065 \pm 0,05$ & $2,01 \pm 0,15$ & - & - & - & $18,8 \pm 0,73$ & $2,45 \pm 0,13$ & $14,40 \pm 0,31$ & $6,65 \pm 0,20$ \\
\cline { 2 - 10 } & $1 \mathrm{~km}$ & $0,115 \pm 0,06$ & $0,045 \pm 0,09$ & $1,70 \pm 0,10$ & - & - & - & $17,0 \pm 0,50$ & $2,77 \pm 0,05$ & $13,05 \pm 0,23$ & $6,70 \pm 0,15$ \\
\hline \multirow{4}{*}{ Ayçiçeği i } & $25 \mathrm{~m}$ & $0,155 \pm 0,04$ & $0,095 \pm 0,01$ & $0,850 \pm 0,03$ & - & - & - & $14,8 \pm 0,41$ & $4,48 \pm 0,13$ & $2,35 \pm 0,20$ & $4,35 \pm 0,03$ \\
\cline { 2 - 10 } & $100 \mathrm{~m}$ & $0,150 \pm 0,06$ & $0,095 \pm 0,03$ & $0,870 \pm 0,05$ & - & - & - & $10,8 \pm 0,44$ & $4,33 \pm 0,10$ & $3,15 \pm 0,10$ & $5,68 \pm 0,25$ \\
\cline { 2 - 10 } & $500 \mathrm{~m}$ & $0,121 \pm 0,01$ & $0,085 \pm 0,06$ & $0,950 \pm 0,15$ & - & - & - & $11,4 \pm 0,53$ & $3,85 \pm 0,13$ & $5,10 \pm 0,30$ & $3,45 \pm 0,20$ \\
\cline { 2 - 10 } & $1 \mathrm{~km}$ & $0,112 \pm 0,03$ & $0,080 \pm 0,04$ & $0,830 \pm 0,10$ & & & & $5,60 \pm 0,49$ & $3,82 \pm 0,15$ & $4,00 \pm 0,23$ & $3,90 \pm 0,14$ \\
\hline
\end{tabular}

Yukarıdaki bilgiler ışı̆̆ında analizi yapılan Antakya-Cilvegözü Karayolu etrafindaki tarım arazilerinin ve bu topraklarda yetişen bitkilerin şuan için önlem gerektiren herhangi bir ağır metal kirliliğine sahip olmadığı görülmektedir. Fe elementi miktarının sınır değerin biraz üzerinde olması, bitkiler için gerekli bir besin elementi olduğu göz önünde bulundurulduğunda herhangi bir sorun teşkil etmediği kabul edilmektedir. Ancak belirli zaman aralıklarında bu tarz çalışmaların tekrar edilerek ağır metal birikiminin zamanla olası değişimi incelenmeli ve gerektiğinde önlemeler alınmalıdır.

\section{KAYNAKLAR}

1. Mater, B., 1998. Toprak Coğrafyası. İstanbul: Çantay Kitabevi.

2. İlkılıç, C., Behcet, R., 2006. Hava Kirliliğinin İnsan Sağlığ ve Çevre Üzerindeki Etkisi. Doğu Anadolu Bölgesi Araştırmaları, 5(1): 66-72.

3. Haktanır, K., Arcak, S., 1998. Çevre Kirliliği. Ankara Üniversitesi, Ziraat Fakültesi, Toprak Bölümü, Ankara. Yayın No: 1503.

4. Li, X., Liu, P.S., 2001. Heavy Metal Contamination of Urban Soils and Street Dusts in Hong Kong. Applied Geohemistry 16: 1361-1368.

5. Karataş, M., Güler, E., Dursun, Ş., Özdemir, C., Argun, M.E., 2007. Konya Ana Tahliye Kanalının Çengilli Bölgesi Tarım Topraklarında ve Buğdayda $\mathrm{Cu}, \mathrm{Cr}$, Ni ve $\mathrm{Pb}$
Derişimlerinin Belirlenmesi. Süleyman Demirel Üniversitesi, Fen Dergisi, Say1 29, 91- 99.

6. Zincirlioğlu, N., 2013. Investigation of the Heavy Metal Contents of Some Agricultural Lands in the Region of Manisa-Akhisar. Ege Üniversitesi, Ziraat Fakültesi Dergisi, 50 (3), 333-339.

7. Özkan, A., Sungur, Ş., Özkan, V., Birses, H., 2017. Heavy Metal Pollution Around International Hatay Airport. Natural and Engineering Sciences an International Journal. Volume 2, No 1, 18-24.

8. Karaçağıl, D., 2013. İstanbul'da Belirlenmiş Sahil Şeritlerinde Toprak Kalitesi ve Ağır Metal Kirliliği, adlı Yüksek Lisans Tezi. Bahçeşehir Üniversitesi, Fen Bilimleri Enstitüsü, Kentsel Sistemler ve Ulaştırma Yönetimi Programı, İstanbul.

9. Knezevic, M., Stankovic, D., Krstic, B., Nikolic, M. S., Dragica, V., 2009. Concentrations of Heavy Metals in Soil and Leaves of Plant Species Paulownia Elongata S.Y.Hu and Paulownia Fortunei Hemsl. African Journal of Biotechnology. 8 (20): 5422-5429.

10. Tao, S.G., Lou, C.Z., Yuan, X.S., Li, W., Ju, Z., Wen, H.L., 2007. Characteristics of Heavy Metal Pollution in Soil and Dust of Urban Parks in Shanghai. Environmental Science, 53: 250-330.

11. Möller, A., Müler, H.W., Abdullah, A., Abdelgawad, G., Uterman, J., 2004. Urban Soil 
Pollution in Damascus Ghovta. Geoderma, 124: 63-71.

12. Sezgin, N., Ozcan, H.K., Demir, G., Nemlioglu, S., Bayat, C., 2003. Determination of Heavy Metal Concentrations in Street Dusts in Istanbul E-5 Highway. Environment International, 29: 973-985.

13. Haktanır, K., Arcak, S., Erpul, G., 1995. Yol Kenarlarındaki Topraklarda Trafikten Kaynaklanan Ağır Metallerin Birikimi. Turkish Journal of Engineering and Environmental Sciences, 19: 423-431.

14. Kacar, B., Katkat, V., Öztürk, Ş., 2013. Vegetable Physiology. Nobel Akademik Yayıncılık Eğitim ve Danışmanlık Ltd. Şti. Ankara. Türkiye.

15. Lindsay, W.L., Norwell, W.A., 1978. Devellopment of DTPA Soil Test Zinc İron, Manganese and Copper. Soil Science Society of American Journal, 42, 421-428.

16. Loeppert, R.H., Hallmark, C.T., Koshy, M.M., 1996. Routine Procedure for Rapid Determination of Soil Carbonates. Soil Science Society of American Journal, 48, 1030-1033.

17. Nelson, D.W., Sommers, L.E., 1982. Total carbon, Organic Carbon, and Organic Matter. In Methods of Soil Analysis, $2^{\text {nd }}$ edn, Edited by: Page, A.L. 539-579. Madison, Wisconsin: American Society of Agronomy. Part 2.

18. Demiralay, İ., 1993. Toprak Fiziksel Analizleri. Atatürk Üniversitesi. Yayın. No: 143. Erzurum. 90-95.

19. Gee, G.W., Hortage, K.H., 1986. Particle- Size Analysis. Methods of Soil Analysis. Part 1. Physical and Minerological Methods Second Edition. Agronomy No: 9, 383-441.

20. Öztürk, M., Bildik, B., 2005. Hayvan Çiftliklerinde Kompost Üretimi. Çevre ve Orman Bakanlığı, Ankara.

21. Anonim, 2005. Resmi Gazete. Toprak Kirliliğinin Kontrolü Yönetmeliği. Say1: 25831, Tarih: 31.05.2005.

22. FAO/WHO Codex Alimentarius International Food Standards Codex Stan-179, 2003. Codex Alimentarius commission. 
